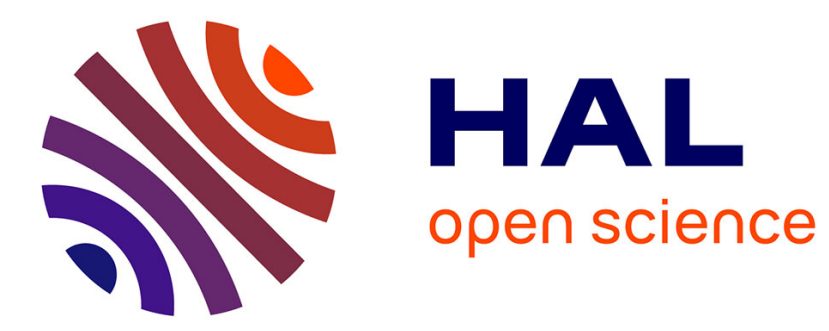

\title{
La profitabilité dans le premier plan d'affaires imprimé en France (1569-1612)
}

\author{
Luc Marco, Robert Noumen
}

\section{To cite this version:}

Luc Marco, Robert Noumen. La profitabilité dans le premier plan d'affaires imprimé en France (15691612). Recherches en sciences de gestion, 2016, 38 (115), pp.149-166. hal-01484413

\section{HAL Id: hal-01484413 \\ https://sorbonne-paris-nord.hal.science/hal-01484413}

Submitted on 7 Mar 2017

HAL is a multi-disciplinary open access archive for the deposit and dissemination of scientific research documents, whether they are published or not. The documents may come from teaching and research institutions in France or abroad, or from public or private research centers.
L'archive ouverte pluridisciplinaire HAL, est destinée au dépôt et à la diffusion de documents scientifiques de niveau recherche, publiés ou non, émanant des établissements d'enseignement et de recherche français ou étrangers, des laboratoires publics ou privés. 
SCIENCES de GESTION Management sciences Ciencias de Gestión

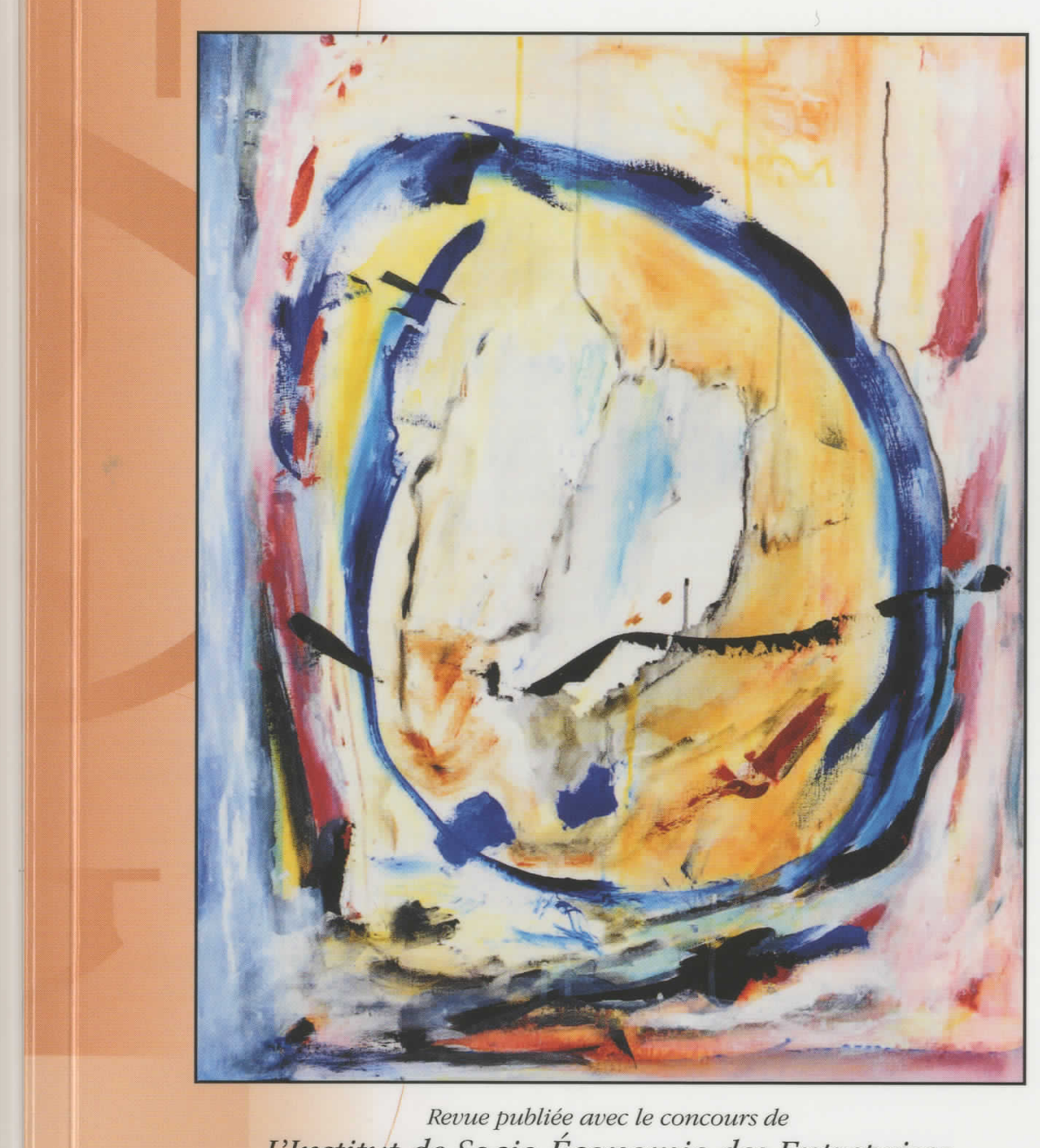

L'Institut de Socio-Économie des Entreprises et des Organisations

№ $115-2016$

ISEOR éditeur 


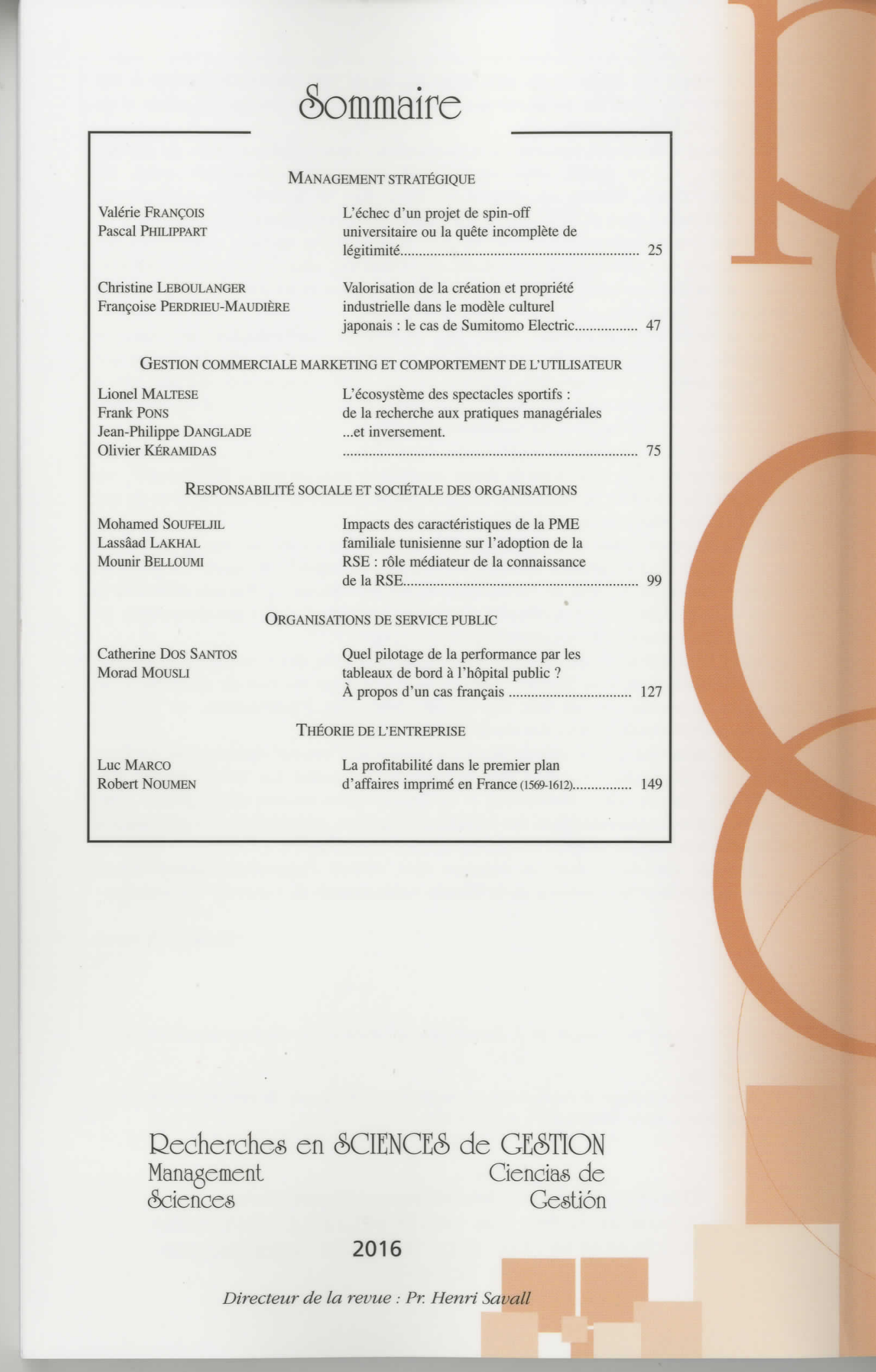


La profitabilité dans le premier plan d'affaires imprimé en France (1569-1612)

Luc Marco

rofesseur émérite

Chercheur au laboratoire CEPN-CNRS

UFR Sciences économiques et gestion

Université Paris 13 Sorbonne Paris Cite (France)

$$
\text { Robert Noumen }
$$

Maître de conférences - HDR

Chercheur au laboratoire CEPN-CNRS

Département GEA de l'IUT de Saint-Denis
Université Paris 13 Sorbonne Paris Cité

Université Paris 13 Sorb
(France)

Quand a été édité le premier plan d'affaires français? Sur quel type d'entreprise portait-il ? Le processus de retour d'investissement etait-il dans le titre? Autant de questions qu' il faut se poser pour faire une histoire des plans d'affaires en France. Et comme ce document est imprime au plus fort de la revolution agricole qui secoue l Europe en cette fin de la Renaissance, il faut aussi charges les produits et les besoins visés? Les profits anoncésts, les réalistes?

Mots-clés : Profitabilite 
Ce livre n'a eu que deux éditions du vivant de l'auteur. En revanche les millésimes ultérieurs, modifiés par les éditeurs successifs, ont e
une diffusion internationale.

\section{a.Un magistrat et avocat de province}

Prudent le Choyselat, dont le nom signifiait en ancien français "le meunier ", est né vers 1530 dans une famille de magistrats. Après des études en droit, il est devenu procureur du roi Charles IX et de la reine Catherine de Médicis (tant que son fils ne fut pas marié en $1570)^{5}$ à Sézanne, petite ville près de Châlons-sur-Marne ${ }^{6}$. II relevait
du gouvernement de Champagne et de Brie sous l'autorite du Bailly du gouvernement de Champagne et de Brie sous l'autorité du Bailly de Meaux, et a dú etre nommé procureur vers 1560 car il avait déjà ce janvier 1561. Son prédécesseur aux Etats généraux d'Orléans en Janonyme, 1546, feuillet evji) parties: un part du roi payée en quatre trimestres pas de souverain gardait souvent le quatrième pour son budget (en ́́ch d'une rente), et une part du baillage payée en nature ou en argent par les clients de son activite d'avocat. C'est à l'occasion de la demande par sa femme, Louise Poullet, du paiement de l'arrière de la part non payée par le roi que nous apprenons qu'il est mort en 1577: « 855 . Sezanne (Brie). Quittance donnée par Louise Poullet, veuve de Prudent Le Choiselat, procureur du roi au Bailliage de Sezanne, des arrerages de rentes qu elle possede sur les Aides de l'élection de Sezanne, pour argent prêté au Roi. 17 octobre 1577 , parchemin.
(Techener, 1972, p. 247)

\section{b.Evolution du texte} Silly aux Etats généraux est reproduit dans de Thou (1742, p. \&-9). 5. Charles IX, devenu roi en 1560 , ne fut majeur qu' en 1563 , il se maria le 26 novembre
1570 et mourut en 1574 . Henri III lui succéda al ors (voir Bordons 15 . comté de Sézanne resta possession da alors (voir Bordonove, 2009) 6. Le comte de Sézanne resta possession du roi de France jusqu'en 1581 ou il passa au
Duc d'Angouleme. En 1566 la ville fut prise par les Huguenots qui la pillerent (Baye, 1875 , p. 3 .

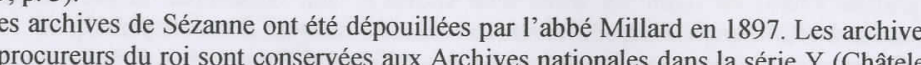
de Paris et preveôté de de l'lle-de--France) dans les cartonn VIIII - Communautét des

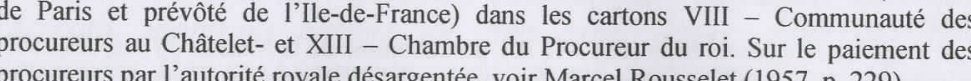

Sortie en 1569, la première édition était pourtant autorisée par la censure royale depuis le 30 mai 1567 . Pourquoi avoir attendu près de deux ans pour éditer le texte? L'évolution de la législation du secteur explique cela. La vente d'œufs était au départ assurée par une
corporation de poulaillers ou vendeurs de volailles (dont les poules, corporation de poulaillers ou vendeurs de volailles (dont les poules,
coqs et poussins). Leurs concurrents étaient les oyers-rôtisseurs qui coqs et poussins). Leurs concurrents étaient les oyers-rôtisseurs qui
vendaient, eux, des oies rôties. En 1509 Louis XII donna le droit aux vendaient, eux, des oies rôties. En 1509 Louis XII donna le droit aux

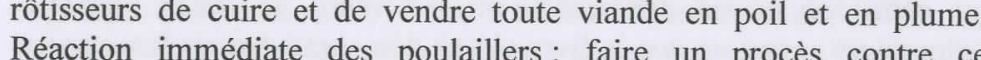
privilège qui leur enlevait tous leurs bénéfices. En 1526 les rôtisseurs font interdire aux poulaillers la vente de viande cuite, ce qui réduisat encore leurs marges. En 1546 le roi François II, excédé par longueur de ce procès, rend cette profession libre. Ce qui n'empêch pas un nouveau procès en 1554, le Parlement de Paris tranchant en deux: la viande crue pour les poulaillers, la cuite pour les rôtisseurs. Enfin, en 1578, ce fut la victoire finale des mechants rôtisseurs : 1 corporation des poulaillers disparait provisoirement.

Le magistrat Le Choyselat a aussi remarqué qu'en 1567 le roi Charles IX a fixe les prix de ces aliments: le plus gros chapon à 7 deniers. Le but était de tenter d'arrêter l'inflation qui arait ate 20 l'économie du pays en créant le corps des Inspecteurs des marchés, chargés de contrôler ces prix sur les marchés français. Notre auteur donc attendu deux ans pour que les critères économiques relatifs à son plan d'affaires soient un peu stabilises. Et comme son projet es adressé à un ami ruiné, il a dû attendre de constater sa déchéance effective découlant de la guerre entre catholiques et protestants, pour publier sa brochure destineès à le remettre en fortune. Selon $\mathrm{La}$ Croix du Maine cite par Huzard (1830, p. 11) l'ouvrage aurait bien été compose en 1567 au pays de Lodunois , puis completé par une page de la formule « \& soixante-neuf " (Le Choyselot, 1569, p. 8 dans notre traduction).

La deuxième édition, datée de 1572, était due au fait que l'accord de la censure courrait sur sept ans à compter du 30 mai 1567 : il fallait utiliser ce droit à temps. Le fait que les caractères utilisés soient usés montre que cette réimpression a été faite à partir de la première composition du texte.

8. Loudun se trouve près de Rochefort: l'imprimeur a êté recommandé à notre auteur 
La troisième édition, datée de 1581, est postérieure à la mort de l'auteur comme nous l'avons vu plus haut. Une édition intermédiair
de 1575 n'a pas été retrouvée.

La quatrième édition datée du 5 octobre 1585 remplace la partie " mesnagement » par l'ajout du morceau de phrase : "qui est le moyen de faire profiter son argent.» Là aussi initiative de l'éditeur qui cette fois-ci est différent : « on les vend, au Palais, par les
Colporteurs », preuve que le livre s'était démocratisé pour être vendu, sous une forme moins luxueuse (petit in-octavo) ${ }^{9}$, aux classes populaires par colportage dans les campagnes et petites villes.

La cinquième édition, datée de 1586, est ressortie en 2011 dans une vente aux enchères chez Binoche et Giquello : "Discours oeconomique, non moins uitle que récreatif. Monstrant comme par le employées, l'on peut tirer par an quatre mille cing cents livres de proffit honneste, Anvers, Francois Garet, (en fit, Lyon, Benoist Rigaud) 1586, in-16.

La sixième édition, datant de 1590-95, a été publiée à Paris par Feury Bourriquant (voir Huzard, 1830, p. 16-17). La sep à Pa édition française, datee d'août 1598, est publiée à Fréjus par le Bon Mesnager. Le titre se termine par le morceau de phrase : " qui est le moyen de faire profter son argent sans usure ». Une reimpression Ces éditions du sud de la France sont contemporaines de l'Edide Nantes qui chasse les protestants vers les régions méridiones de Max Weber a montré que l'esprit protestant était plus favorable aux ans d'affaires que l'esprit catholique, moins entreprenant (Besnard, 1970).

Une septième édition, datée du 15 mars 1612, a été publiée par Martin Le Menestrier à Rouen. Dans le titre le mot "profiter » est remplacé par " profier »! C est une habile contrefaçon de l'edition de 1572 mais elle reste, selon Huzard (1830, p. 22) comme la plus belle edition avec contrefaçon.

Concernant le format des livres de cette époque, se référer aux gabarits dans le livre
PRANCE (1569-1612)

Une huitième édition, parue en 1800 (an IX de la République), est publiée par Cussac, imprimetr-libraire à Paris. C'est une contrefaçon de l'édition précédente de 1612. Elle a été vendue par la maison Techener jusqu'en 1835 à un prix compris entre 5 et 50 francs de l'époque.

Au total ces huit éditions françaises, plus les deux éditions anglaises (1577 et 1580$)^{10}$ et l'édition allemande de 1615, ont tirage total de ces onze éditions a dû dépasser les 10.000 exemplaires, d'où la sortie régulière en vente aux enchères de ces curieuses brochures.

\section{.Influence du livre jusqu'en 1650}

Deux ans après la première édition, le poète François De Belleforest, qui avait donné le poème introductif à Le Choyselat,
traduit le livre de l'agronome italien Augustin Gallo (1571). Dans le titre on trouve le mot "mesnagerie » et, à la place des profits honnêtes, des plaisirs honnêtes! Même éditeur ${ }^{11}$, même public : la filiation est évidente. Mais celui-ci ne cite pas Le Choyselat dans sa liste des grands auteurs en la matière : il ne donne

Caton, Columelle, Crescens, Varron et Constantin.

Un auteur important a été influencé par notre homme : Olivier de
res (1600) qui est considéré comme le "père de l'agriculture Serres (1600) qui est considéré comme le "père. de l'agriculture
française ». Appelé par Henri IV à Paris, il introduisit les mûriers française ». Appelé par Henri commandé par le Roi en 1600 et imprimé à plusieurs milliers d'exemplaires pour le développement de l'économie rurale.

Il étudie très rapidement le problème des poulaillers en deux endroits de son livre. Dans le cinquième «lieu », intitulé «De la conduicte du poulailler, du Colombier, de la Garenne, de l'Estang, du Ruscher et des Vers-ä-Soye ", il presente une classification de la volaille qu'il divise en deux : la terrestre et l'aquatique (p. 553). La terrestre se répartit en 8 espèces : la poule commune ou domestique,

10. La premiere de 1577 est encore en vente par les éditions Early English Books
Online, dans la série « Early History of Industry \& Science ». La seconde n'est qu' une 1. Sur cet éditeur parisien catholique, né en 1533 et mort en 1584 , voir l'article 
race française très ancienne; la poule d'Inde ou Méléagrides, race récemment importée ; les Gélinotes ou faisans de Numidie; les poules d'eau; les hérons; les Ortardes; les Hallebrans; et les Aigrettes (p. avec les chapons qui les distraient (p. 557). lI utilise teons " profitable » très souvent.

L'expression « entreprise profitable » est en effet très utilisée à cette époque. Les premières utilisations du mot « entreprise » (au sen de projet) dans les titres d'ouvrages sont, d'après nos recherches : $D e$ l'entreprise et voyage de Naples d'Octavien de Saint-Gelais (vers 1530), Discours sur la chrétienne et louable entreprise, de Scipion de Rogres (1572) et La valeureuse entreprise, sur la ville de Han, aux frontières de la Picardie, par le duc de Bullon (1595). En fevrier 1607 la déclaration du roi relative au desséchement des marais indique : public, nous recognoissons qu'il fout un grand fonds profitable au une continuelle assistance de persm ands de deniers, et affaires (...) » (Isambert, 1829, p. 314-315) ${ }^{12}$. En 1616 Théodore de Bry a cette formule habile: " $y$ balancant les profits avec les dangers » (p. 35). Enfin le lien entre les profits et l'entreprise est fait en 1631 par Pierre Mathieu : « ... grands profits de cette entreprise, ils offroient cent mille francs, pour aider à payer les frais de guerre. » (p. 191). Notre auteur visait les profits maximaux; voyons comment concevait la profitabilité.

\section{2. - Calcul de la profitabilité de l'entreprise projetée}

Dans ce plan d'affaires le promoteur présente les éléments de son marché en identifiant les besoins de sa clientèle potentielle dans le
cadre d'une logistique aval, puis dans une démarche de logistique interne. Il va retenir plusieurs éléments : la difficulté que rencontrera l'entreprise face aux maladies des poules et des coqs, les
conséquences de cette situation face à la physionomie des coûts sanitaires, et les bénéfices escomptés par l'entreprise.

a.Les besoins des clients

12. Déjà le 16 avril 1601 le roi Henri IV édicte une loi intitulée : Traité VII du Monarchies, avec la Commission du Roy à ses fins, 17 pages.
Ceux-ci sont assez simples. Il s'agit de préciser la qualité des produits apprecies par les consommateurs. On y affirme la localisation du marché parisien à travers ses contraintes : adaptabilití du produit, Tu vendras ou feras vendre chacun couf frais aisément six derniers Tu vendras ou feras vendre chacun ouf frais aisément six derniers de Paris qui bailleront librement \& volontiers pour chacun ceuf un carolus s ${ }^{13}$ s'ils étaient assurés qu'ils fuissent frais, de jour à autre, que je dirais deux milles personnes, je penserais ne rien avancer contre la vérité. $\|^{14}$

En plus dans cet ouvrage la représentation du besoin se confond avec la notion de bien-être de l'époque, les habitudes de consommation et les exigences de la clientele etant visees à la fois dans leur dimension subjective, culturelle et religieuse. C'est par determination du besoin pour combler une demande ou une absence par nécessité sú le msuivantes: "Combien y at-il de gentilshomms \& damoiselles qui désireraient sur lo matin l'euffrais? Combien y a t-il de bourgeois \& bourgeoises touchés de ce même désir? Combie de personnes vieilles ennuyees de chair souhaitent l ceuffrais pour diner? Car tu n'ignores pas que l'cuf de sa nature est prompt à nourrir, \& nourrit délicatement, \&cause le bon sang ... ALBERT le grand dit, qu il répare autant de bon sang en la personne, que

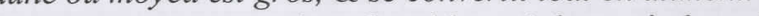

Nous résumons dans le tableau 1 les trois hypothèses du plan d'affaires émises par Le Choyselat. Il fait deux hypothèses : l'une haute, lautre basse. Nous a vispellerons "hypothèse normale ". Poor faciliter les calculs, nous avons arrondi les sommes en livres tournois, pour ensuite remettre les sommes selon les différentes gradations de la monnaie de l'époque : en livres tournois, sols, deniers.
13. Monnaie ancienne valant dix deniers tournois.
14. Prudent Le Choyselat, 2015, p. 23 . 


\begin{tabular}{|c|c|c|}
\hline Poste & \begin{tabular}{|l} 
Montant \\
en Livres
\end{tabular} & $\begin{array}{l}\text { Commentaire } \\
\text { (l'année dure } 365 \text { jours) }\end{array}$ \\
\hline Investissement de départ............ & & Pour financer les coûts fixes. \\
\hline 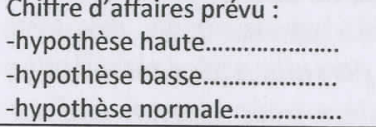 & $\begin{array}{l}7300,0 \\
5475,0 \\
6387,5\end{array}$ & 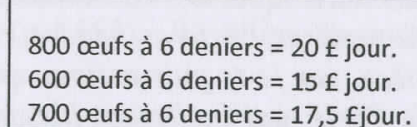 \\
\hline $\begin{array}{l}\text { Coût fixes: } \\
\text { - location ferme sud de Paris. } \\
\text {-achat de } 1200 \text { poules........... } \\
\text {-achat de } 120 \text { cosas............ } \\
\text { - location d'un ânier............. }\end{array}$ & \begin{tabular}{|l|}
500 \\
61 \\
300 \\
48 \\
91 \\
\end{tabular} & $\begin{array}{l}\text { Investissement de départ } \\
365 \text { liv. dans le texte (27, rectifié) } \\
5 \text { sols } \times 1200=300 \text { livres } \\
120 \times 8 \text { sols }=48 \text { livres } \\
7 \text { lives et } 11,66 \text { sols par mois }{ }^{15}\end{array}$ \\
\hline 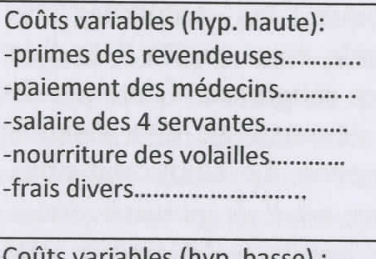 & \begin{tabular}{|l|}
2203 \\
364 \\
260 \\
365 \\
787,4 \\
426,6
\end{tabular} & 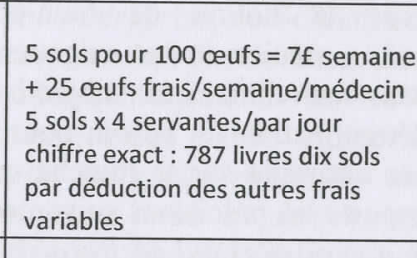 \\
\hline 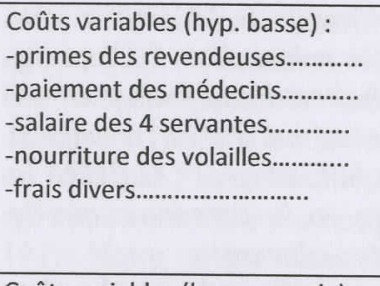 & $\begin{array}{l}1101,5 \\
182 \\
130 \\
182,5 \\
393,7 \\
213,3\end{array}$ & $\begin{array}{l}2,5 \text { sols pour } 100 \text { œuff }=3,5 \\
\text { f/semm. } \\
\text { Plus } 13 \text { œufs frais par semaine } \\
2,5 \text { sols } 4 \text { 4 esvantes/par jour } \\
\text { Chifffre exact : } 393 \text { livres six sols } \\
\text { Par déduction des autres frais } \\
\text { variables }\end{array}$ \\
\hline 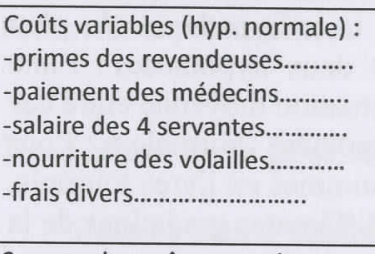 & \begin{tabular}{|l|}
1652,25 \\
273 \\
195 \\
273,75 \\
590,55 \\
319,95 \\
\end{tabular} & $\begin{array}{l}\text { 3,75 sols } / 100 \text { cuufs }=3,5 \mathrm{f} / \mathrm{sem} \text {. } \\
\text { Plus } 19 \text { ouufs frais par semaine } \\
3,75 \text { olls } 44 \text { servantes/par jour } \\
\text { Chiffre exact } 5990 \text { livres huit sols } \\
\text { Par déduction des autres frais } \\
\text { variables }\end{array}$ \\
\hline 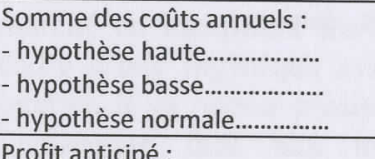 & $\begin{array}{l}2703 \\
1601,5 \\
2152,25 \\
\end{array}$ & $\begin{array}{l}500+2203 \text { (plus } 15 \text { sols) } \\
500+1101,5 \\
500+1652,25\end{array}$ \\
\hline 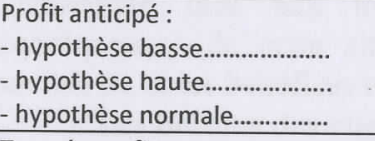 & $\begin{array}{l}3447 \\
4596 \\
4021,5\end{array}$ & 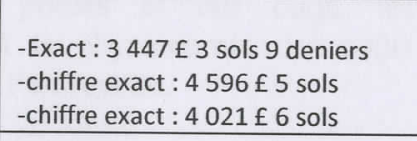 \\
\hline $\begin{array}{l}\text { Taux de profit: } \\
\text { - hypothèse bas: }\end{array}$ & & \\
\hline
\end{tabular}
15. Nous avons considere que la nourriture des ânes ( 91,5 lives) était avancée p
lânier, ce qui constitue la seule avance que nous avon

\begin{tabular}{|c|c|c|}
\hline $\begin{array}{l}\text { - hypothèse haute........................... } \\
\text {-hypothèse normale.......... }\end{array}$ & $\begin{array}{l}819,2 \% \\
704,3 \%\end{array}$ & $\begin{array}{l}\text { rapport entre le résultat net et les } \\
\text { investissements. }\end{array}$ \\
\hline 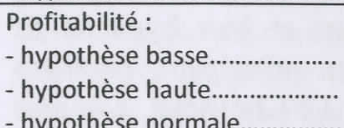 & $\begin{array}{l}63,0 \% \\
63,0 \% \\
630 \%\end{array}$ & $\begin{array}{l}\text { C'est ici le rapport entre le résultat } \\
\text { net et le chiffre d'affaires. II est } \\
\text { identique pour les } 3 \text { hypootheseses. }\end{array}$ \\
\hline
\end{tabular}

NB : Une livre tournois vaut 20 sols ou 240 denies.

Ce marché hautement profitable ( $63 \%$ du chiffre d'affaires se transforme en résultat net) va dépendre de différents facteurs physiologiques, des conditions climatiques, environnementales et des contexte global, s'il n'est pas maîtrisé, peut être à l'origine des maladies sources de dépenses supplémentaires pour le projet : les frais sanitaires representent $28,4 \%$ du total des coûts variables. Et faudrait compter avec le renouvellement du poulailler qui est un
matériel qui pourrait s'amortir. Le Choyselat, lui, insiste sur le risque sanitaire.

\section{b.Les maladies des poules et des coqs}

Le modèle économique développé dans Le Choyselat nous propose un projet soumis à une contrainte centrale qui est le risque de la maladie des poules et cogs. Celle-ci participe à la détérioration de l'extrant source d'une qualité du produit non conforme à l'idée de départ, aux besoins de la clientèle visée car désormais le produit ne respectera pas un niveau de valeur qui puisse le rendre profitable :
est cassé, flasque, immangeable (au bout d'une semaine environ).

est casse, flasque, immangeable (au bout d' the semaine environ).

Il s'agit, pour le créateur d'entreprise, d'un risque technique qui porte sur la maitrise de la production des poules et des coqs dont la étant dangereux pour le projet, Le Choyselat y prêtait une attentio particulière en respectant une "procédure originale " d'identification de la maladie allant de sa localisation à la réponse adéquate à apporter, en passant par un inventaire des réponses possibles.

C'est pourquoi, pour atteindre un objectif de rentabilité, les fermes doivent lutter par la prévention contre les maladies qui frappent les betes car les oiseaux malades sont improductís. Face à

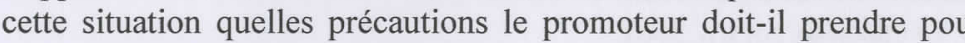
limiter l'introduction et la diffusion des maladies ?

16. Pour un calcul du plan d'affaires sur trois années pleines, voir notre article dans 
Cette situation met en évidence le caractè̀e coûteux des méthodes de contrôle sanitaire, notamment en ce qui concerne propreté des poulaillers à travers leur gestion et leur hygiène. Et (internes et externes) affectant la volaille. Pour Le Choyselo parasites commune maladie est la pépie, ou pituite, qui cost maladie à la la plus le bout de laquelle s'endurcit en façon d'un cartilage \& perd la poule le goût du boire \& du manger, \& infecte les poules, principalement entre la moisson \& les vendanges. " "Il parle aüssi de la gratelle, maladie due aux parasites des paniers de ponte et de couvage (p. 20 de notre traduction).

Ces coûts sanitaires ${ }^{18}$ sont en réalité dans le processus de la réalisation du projet des coûts récurrents. Ils grèvent profondément la fois les flux nets dans le cadre de l'éaboration d'un délai de valeur actuelle nette ayan un pas calculé à l'époque. Pour Ie Choyselat les choses sort phos simples car il dit: " déduisons la dépense \& ce qui restera nous l'appellerons profit : car les jurisconsultes disent que nous n'appelons point fruit ou profit sinon ce qui reste après que la dépense est déduite, comme Papinian Jurisconsulte très célèbre l'a bien défini a vingt-quatrième livre des Pandectes, sous le titre de la répétition de la dot, après le mariage dissous. " (p. 27). Cette approche très pragmatique s'explique par le fait qu il veut vendre plus cher que 1 prix de marché imposé par le Roi, ou que le prix entre particuliers

\section{c.Les bénéfices et la rentabilité}

Dans Le Choyselat, les dépenses (ou sorties de fonds) sont en réalité composées des coûts initiaux (investissements effectués notamment pour la construction du poulailler) et des coûts récurrents, surtout les frais inhérents aux soins de la volaille et à la logistique d'acheminement sur le marché : "Pour la dépense des anes, encore qu'il croisse ordinairement force chardons ès environs de telles
métairies, nous jetterons cinq sols par jour, revenant à quatre-vingt 17. Le Choyselat, 2015, p. 30-31

18. Le prix paye aux medcons etait élevé car le nombre de praticiens officiels était for 19. Les professionnels devaient vendre sur an mo tandis que les quidams qui avaient un enclos pouvaient vendre directement aus
Cinq sols par an. Et pour l'ânier, duquel on peut tirer quelque autre service, après qu'il aura conduit ses ânes au port des jour, \& par an quatre-vingts onze livres cing sols, lequel néanmoins avertiras de ne tant changer ses ânes, qu'ils ne puissent encore bie porter, ou la patience, ou le murmure qui se trouve ès convents 'aucuns moines non réformés $s^{20}$. " Quant aux entrées de fonds, elles que d'un " produit dérivé « qui est ici la vente des poules et des cor en surnombre. Il s'agit aussi d'avantages financiers intangibles car taille croissante du cheptel garantit un cash-flow régulier et sîr.

En tenant compte des flux d'entrées et sorties dans le projet,

est possible de déterminer le délai du retour sur investissement. Le seuil de rentabilité se situe au bout de 35,8 jours (716/20) et donc au bout de la vente de 28640 œufs $(800$ x 35,8). Au-delà de cette limite entreprise est bien rentable puisqu' ici le capital est assimilé aux coûts fixes. Un mois et six jours après la creation de l'entreprise c'est abtient 417 jours $(626 / 15)$ et 25020 inf $(600 \times 417)$. l'hypothese normale on obtient 38,57 jours $(675 / 17,5)$, 041,7$)$. Pour 'eufs $(700 \times 38,57)^{21}$. Donc l'entreprise est rentable en moins d' mois et demi dans tous les cas de figure !

C'est pourquoi le calcul du bénéfice est intimement lié à différence entre le produit de vente et le coût de tous les éléments qui participent à la qualité du produit final. C'est-à-dire ici les œufs qui sont mis à la vente (hors ceux consacrès à la reproduction du chepte par couvage des poussins). Le Choyselat nous dit: "Maintenant reste à te faire connaitre le profit qui reviendra par chacun jour de ta somme: tous frais faits, depenses déduites, la pension de ta maison pédecins, les revendeuses contentées, la dépense des anes \& âniers précomptée \& la mangeaille: car sur la vente tes mufs ne se prendra imposition ou gabelle comme se faisait du temps de Jea Ducas, ... $)^{22}$

Les taux de profits extraordinaires ont dû faire leur effet dans les campagnes du temps, mais c'était trop beau pour être vrai et Jean De
La Fontaine en tirera la fable de la poule aux cufs d'or ! Les louanges

\section{Le Choyselat, 2015, p. 26 .}

21. Calcul : charges fixes/taux de marge sur coûts variables. Soit, pour l'hypothèse
haute $500069,82 \%=716,13 ;$ pour l'hypothese basse : $500 / 79,88 \%=625,94$; et pour P'hypothèse normale : $500 / 74$,
22. Le Choyselat, 2015, p. 22 
des partisans de Le Choyselat étaient très naïves et les dubitatifs n'on pas laissé de trace écrite selon nos recherches. Les principales critiques ne vinrent qua a partir dn moment ou les cours d agriculture laviculture (Thouin, 1809).

\section{d.Critiques du plan d'affaires le choyselien au XIX ${ }^{e}$ siècle}

En novembre 1845 un spécialiste de l'élevage des poules critique le livre de notre auteur (Loiseleur-Deslongchamps, 1845, p. 469-473) sur les quatre points suivants :

${ }^{\circ}$ ) Le Choyselat a surévalué la production d'œufs par ses poules (800 œufs par jour!) ce qui est irstiste car il n'a pas tenu comple des saisons et des races avicoles; cette erreur devrait réduire du tiers ou de moitié le revenu supposé par notre auteur (p. 470).

$2^{\circ}$ ) Même problème du côté de la vente des œufs : le prix demandé est irrealiste car la demande n'est pas rare pendant la belle saison et on a diminué des $5 / 6^{\circ}$ pour avoir une évaluation raisonnable (p. 471).

$3^{\circ}$ ) La nourriture des poules (2 setiers parisis d'orge, d'avoine et de vesceron) était alors beaucoup trop chère : il aurait fallu donner aliment moins coûteux (son détrempé) pour réduire ce coût de moitié.

$4^{\circ}$ ) Les pertes dues aux maladies des poules et à leur décès inopin n'ont pas aussi été prises en compte par notre auteur, qui ne provisionne pas une somme précise à cet effet (p. 472)

D'où la conclusion de ce spécialiste : «je ne pense pas que ce livre ait jamais pu être un ouvrage utile et que jamais personne ait pu mettre en pratique pour se faire un revenu certain. » (Ibidem). Mais il lui trouve encore un certain intérêt car le prix des poules était nettement plus bas en 1569 qu en 1845, quand on se base sur la valeur du marc d'argent pour faire la conversion: 1 marc permettait alors d'acheter 68 poules, tandis qu au milieu du XIX siecle il faut 4 marcs Pourtact 1852 une veuse (p. 47)

récemment retirée à campagne, à Belair sur la commune de Charny dans l'Yonne, mit en application le plan d'affaires de notre auteur; c'était madame (Gayot, 1864). Partant d'un cheptel initial de 40 poules, elle obtint 作ces avec l'aide des ménagères du coin (p. 44), puis atteignit le chiffre choyselien de 1.200 poules en 2 mois et 6 jours. Les restiltats firent bons et il etait donc possible dapplisuer le plan d'affaires de notre auteur en utilisant les méthodes modernes
d'élevage avicole (p. 186).

\section{Conclusion}

De par son titre, l'opuscule de Le Choyselat fait partie de 'économie domestique et rustique : il est le successeur de $\mathrm{Y}$ 'anglais italien Tarello (1567). N'étant pas le premier à introduire le mot "profit" dans son titre, il va cependant plus loin que ses prédécesseurs en mettant l'accent sur le retour d'investissement: mettre 500 livres tournois pour en gagner 4.500 était une folle promesse pour temps fous : mente les heguenots pourtant portés au rand commerce ont dû trouver cette performance extroording a (Daussy, 2014

u vu de son contenu il relève d'un champ plus large: les sciences agronomiques et véterinaires. Dans le premier secteur il forts, des points faibles, état des charges prévisionnelles, deux hypothèses de profit, annonce alléchante d'une forte profitabilité à terme. Son ami ne pouvait qu'être ravi à défaut de redevenir riche immédiatement. Dans le second champ on doit à Jean-Baptiste Huzard (1755-1838) d'avoir remis toute la lumière sur ce livre et de l'avoir

Enfin, dans le champ des sciences de gestion (Marco, 2016), il
conserve dans fait figure de précurseur car il temoigne de l'apparition du «nouvel esprit commercial apparu dans lagriculture - en liaison avec les littérature qui sherchait à diffuser ces nouvelles méthodes de production généralement ànnus sous le nom de Révolution Agricole.) (Schumpeter, 1954, p. 223-224). En mettant dans son titre à la fois le mot « économique » et la notion même du plan d'affaires

23. Traduit en français en 1564 par son gendre Jean Liébault sous le titre de la Maison

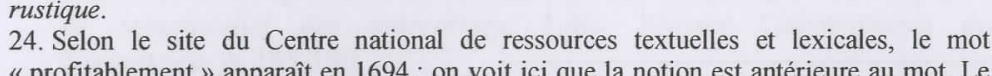
premier texte francais où le mot «profitable » est dans le titre date de 1486: Le livre ntitule internelle consolation très utile et proffitable, traduction du latin par Tho 
basé sur la recherche du profit maximal, il a révolutionné la manière de rédiger des prospectus d'affaires à venir : être précis et ambitieux En retenant un ecart de $25 \%$ entre a hypothese basse et l'hypothèse il doit être lu et relu à la lumière de nos connaissances actuetles sur l'économie privée du seizième siècle ${ }^{25}$ L'art de la profitabilié a dor précédé les méthodes de calcul de la rentabilité (Slywotzky, 2002).

\section{RÉFÉRENCES BIBLIOGRAPHIQUES}

ANONYME, Les coustumes et statutz particuliers de la plupart des bailliages, DE J. de, Sézanne, d'apre

Imprimerie de A. Patoux, Sézanne, 1875 Armand Colin, Paris, 1970 . 3, Franz Steiner Verlag Strttgy and $M^{2}$.

la controverse post-webérienne onné, Pygmalion, Paris, 2009. BULLON, duc de, La Valeureuse entreprise, sur la ville de Han, aux frontières de la Picardie, Albert Henry, La Haye, 1595.

CALLEBAT L. dir., Histoire du médecin, Flammarion, Paris, 1999.

choses rustiques, traduits par Coude Cottereau, Kerver, Paris, 1553. CRESCENS P. de, Le livre de prouffitz champestres et ruraulx, Veuve Le Noir,

Paris, 1521 (écrit vers 1320).
DaussY H., Le parti huguenot, chronique d'une désillusion (1557-1572),

lyonnais du XVImerce et l'industrie de la soie (d'après des inventaires professeur René Gonnard, L, LDDJ, Paris, 1946, p. 81-88.

EDWARDS J.R., A History of Financial Accounting, Routledge, London, 1989. EsTIENNE Ch. [dit Stephanus] (1554) L'Agriculture et maison rustique de M. Charles Estienne docteur en médecine. En laquelle est contenu tout ce qui
peut estre requis, pour bastir maison champestre, nourrir et médeciner

25. Sur la manière de gérer les entreprises industrielles de l'époque, voir la contribution
de Roger Doucet (1946) II faudrait retrouver des inventaires de poulailers dens les archives notariales pour aller plus bon

PRANCE (1569-1612)

bestiail et volaille de toutes sortes..., J. Du Puis, Paris, 1554, réédité avec Jean Liebault en 1578

TA. The Boke of Husbandry. Here begynneth a newe tracte moos profytable for all husbande men: and very frutefell for all other persons to

agriculture, et hosnnetes plaisirs qu' on reço en la mesnagerie des champs, pratiquez \& experimentez tant par autheur qu' autres experts en ladicte science, divisez en XX journées, par 1571 (1 $1^{\text {ter }}$ édition italienne : 1566).

Mricole de la Maison rustique, Paris, GerVaIS P., Y. LEMARChAND \& M. MARGairaz ed., Merchants and Profit in the Age of Commerce, London, Routledge, 2014.

. business plans, Thèse en sociologie, Université de Toulouse 1, 2010.

HUZARD J.-B., Notice analytique et bibliographique de l'ouvrage de Prudent Le Choyselat sur les avantages que l'on peut retirer des poules, $\mathrm{M}^{\mathrm{me}}$

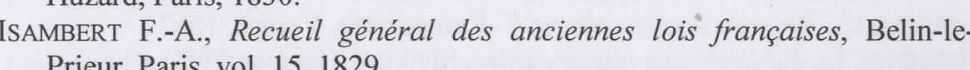
Choysejat P. Diseous

en moins utile que récréatis. enq cens livres, pour une fois employées, l'on peut Chesneau Paris 1569 , réd cent livres de profit honnête, Nicolas Chercis CHOYSELAT P. A Discourse of Housbandrie no lesse proftat

delectable.... London, Myles Jenynges, 1577, traduction moderne et préface par H.A.D. Neville, Université de Reading, 1951

LE CHOYSELAT P., Newe und Nutzbahre Kunst in Hausshaltung, auss dem Frantzösischen in Unsere Teulsche Mutter. Spache Transvertit und OER R, Robert Loder's Farm Accounts $1610-16$

Historical Society, London, 1936. "en-Dis. d'agriculture, d'économie rurale et domestique, novembre, 1845, p. 456ARCO L. "Le Choyselat, fondateur des plans d'affaires à la fin du XV" siècle en France: son dossier financier complet $),$ Revue d'Histoire et de

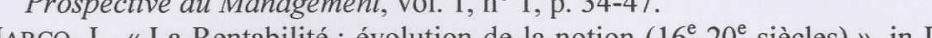

MARCO, L. « La Rer la comptabilité des entreprises, Lille, Presses Universitaires de Septentrion, p. 185-190

MATHEU P., Histoire de France sous les règnes de François $1^{e r}$ à Louis XIII Veuve Nicolas Buon, Paris, 1631 
MiLLARD A., Histoire de Sézanne, Imprimerie A. Patoux, Sézanne, 1897. historives Chamat des livres, notions pratiques suivies de recherches Rataut L., "Nicolas Chesneau, Catholic Printer in Paris during the Frenc Wars of Religion", The Historical Journal, vol. 52, n 1, 2009, p. 23-41. OGRES, Scipion de, Discours sur la chrétienne \& louable entreprise de haut \& puissant Prince Charles de Lorraine, Marquis du Maine, contre It ROUSSELET M. Histoire de la magistrat

政 SAINT-Gelals O. de, Le Vergier

. SCHUMPETER J., Histoire de l'analyse économique, Gallimard, Paris, 1954

SERRES 0. de, Le théd
Arles, $1600,2001$.

SLYWOTZKY A. The Art of Profitability, New York, Hachette UK, 2002. SULLY, Les oeconomies royales, Librairie C. Klincksieck, Paris, 2 volumes

TARELLO C., Ricordo di agricoltura, F. Rampazetto, Venise, 1567. THou J.-A. de, Histoire universelle, Jean-Louis Brandmuller, Bâle, 1742, t. 3 , (période 1560-1567).

THounN dir., "Poulailler, poule ", Nouveau cours complet d'agriculture UNGER R. W., Beer in the Middle Ages and the

Pennsylvania Press, Philadelphia, 2013. for small wine grape growers: evidence from a comparative study WARD J. R. "The Profitability of Sugar Planting in the British West Indies, 1650-1834." The Economic History Review, ns 31.2 (1978): 197-213.
.

\section{Contents}

STRATEGIC MANAGEMENT

Valérie FRANÇOIS

Pascal PHILIPPART

A university spin-off launc Ailure or the uncompleted

Christine LEBOULANGER Françoise PERDRIEU-

Upgrading creation and Industrial property in
Japanese cultural model: the case of Sumitom Electric $\ldots \cdots \cdots \cdots \cdots$

COMMERCIAL MANAGEMENT MARKETING AND BEHAVIOR OF USERS

Lionel MALTESE

Frank PONS

Jean-Philippe DANGLADE

Ecosystem of Entertainmen

Olivier KERRAMIDAS

port: Inventory on the

skills..................... ORGANIZATIONS

$\begin{array}{ll}\text { Mohamed SOUFELJIL } & \text { Impacts of the } \\ \text { Lassâad LAKHAL } & \text { characteristics of the } \\ \text { Mounir BELLOUMI } & \text { Tunisian family business on } \\ & \text { the adoption of CSR } \\ & \text { mediator role of knowledge }\end{array}$

mediator role of of CSR

of CSR .................................

\section{ORGANIZATION OF PUBLIC UTILITIES}

Catherine DOS SANTOS Morad MOUSLI

Performance managemen using balanced scorecards in public hospitals. A French 